\title{
Modeling and Simulation of Anti-Skid Control System of Railway Vehicle in Curved Track
}

\author{
Pacifique Turabimana $^{1 *}\left(\mathbb{D}\right.$, Sosthene Kazima ${ }^{1}\left(\mathbb{D}\right.$, Josee Musabyimana ${ }^{2}(\mathbb{C})$ \\ 'Rwanda Polytechnic/ IPRC Gishari \\ 2University of Rwanda/ College of Science and Technology
}

\begin{abstract}
The slipping of railway vehicle wheels during curve negotiation has been always a major problem in railway transportation. One of the causes of these slippages is predicted to be the lack of proper curve radius which incites high creepages. The creepages cause improper wheel rail interaction during curve negotiation. Most of the light rail transit system, with condensed population, there is a huge demand which increases the railway vehicles' weight to the maximum. However, this weight is again expected to have additional effects on the wheelset slipping when negotiating the curve or braking on a gradient curvature. Therefore, the aim of this paper is to model the anti-skid control of a railway vehicle in curved track operating in two instances: when the train is braking on a gradient curve and when the train is negotiating a curved track. To achieve this objective, the lateral dynamics equations of motion of the wheelset have been solved to predict the yaw angle and lateral displacements as well as their velocities. These quantities are used to calculate the creepages and creep forces. In return, they are input to the control model to limit the skidding. Computer simulations using MATLAB/ Simulink have been carried out to assess the feasibility of the control method. The results have used to design proper control systems that the rail network in congested environment are able to use. Antiskid control offers other benefits such as increasing the lateral comfort by reducing lateral forces and limiting noises generated by skidding in curvatures.
\end{abstract}

Key words: Anti-skidding, creepages, curved track, braking system, wheelset dynamics

\section{INTRODUCTION}

Most railway industries and researchers have focused on railway vehicle stability, reduction of derailment, travel comfort \& safety of passengers. Therefore, it is also meaningful and necessary to include an active safety system during braking system operation with railway vehicle negotiating a curved track. The railway vehicles negotiate a curve itself by wheels' profile which is called self-steering. The problems of wheelset hunting and curving ability by self-steering mechanism persist[1]. When braking force applied to the wheel exceeds a critical value, which depends on the wheel-rail adhesion contact, the sliding or locking up of the wheels on the rail occurs. However, sliding of the wheels on the curved track in both longitudinal and lateral directions can result in increased braking distance, more vibration and damages to the wheels and rails or worse still it can lead to derailment. Railway vehicle brake is an important active safety system where its kinetic energy is converted into heat energy to slow down a vehicle in motion or to stop it as it is needed[2]. However, many different studies were done to control the skidding or sliding during braking, with the different aspects. Therefore, control the dynamic effect of the wheel on rail on straight track based to the different contact angles have performed by many researchers but hunting motion still be a problem. During curves negotiation the problem of hunting replaced by sliding which is more danger for safety issue [3], developed the control system to optimize the critical speed of railway vehicle moving in curved track relative to the high speed. The system based to rolling motion from centrifugal force, radius of curve, rail parameters and the maximum speed to control the derailment angle in the analytical quasi static manner. There was wheel sliding from the attack angle including both lateral and longitudinal, no comfort for passenger at speed below to the maximum and the change of friction coefficient and braking distance increases.

The developed of Anti-slip Control by [4] was based to the mechatronic approach of the traction motors. The detection of slip was done by axle sensor and speed sensor. The Karman filter and pass filter detect the dynamics of the wheelset while brake is applied. At the contact patch between rail and 
wheel there are multiple intersected forces from different direction. However, all of them are affected by creep forces, sliding or creepages and braking forces at that point. The tractive force to overcome the other resistance forces should be continuously and enough as possible. This technique controls the detected slip and adhesion force and it doesn't consider the forward train speed and the slip ratio. There is no big different for the working principle and the result of the technique with the role of the sanding system of the train. Due to a train is braked, the low adhesion manifests as wheel slip where the wheelset is rotating at a lower velocity (speed) than the forward speed of the train. However, in curved track the right wheel and left wheels have different speed from the different chord. The wheel move on the outer rail of the curve needs to move fast to catch up the other wheel at the same wheelset which moves on the inner rail of the curve. The most extreme example of this is where the wheel stops rotating altogether (wheel slide) while the train is still moving and can result in a "wheel flat" caused by the softer wheel still being abraded away by the harder rail steel[5]. The other disadvantageous is that the system is implicated to the powered wheels and is designed to control it during straight curve. During the application of brakes on the wheels there is locked up of the wheels when the wheels torque produced by the adhesive force is not enough to overcome the braking torque generated by the brake force in braking system[6]. Due to the train negotiating a curvature, the slippage is different to the straight track. The longitudinal sliding causes more wheelset vibrations and overheating between wheel and rail which also damage a track line. There is also a lateral sliding caused by shear stress of wheel on a rail and the lateral displacement of a wheelset from the yaw angle and rolling angle. In curved track, the outer wheels rotate differently to the inner wheels and have different contact angles which can cause the unstable of the railway vehicle to the accident of the vehicle by wheel flange climbing of the inner wheel and unbalanced of centrifugal forces[7].

The control system that could control the adhesion/ anti-skid without considering the braking force in curved railroads was developed with the help of dSPACE system by[8]. The mounted sensors on the two axles were produced the encoder signals, the detection axle rotation signals are transmitted to the dSPACE controller as the main input of the system to function. Therefore, its performance and effective were not verified to the successful of anti-skid control. By[9], the friction in curved track was analyzed. The contact area is elliptic or linear at both straight and curved track according to the position of the wheelset on track[10]. However, on curvature there is a forced contact of a wheel flange to the rail head gauge corner by the lateral force and the normal contact of wheel tread and the rail head. The motion of wheel on rail depends on the friction power between them and it is proportional to the longitudinal, lateral and spinning creeps from the different creep forces. However, the friction power is controlled separately on the right and left wheels of the wheelset. It depends to the increased or decreased of the forward speed of the vehicle and the radius of curvature. It is not easy to solve the problem of skidding completely because they have overlooked the changing of the equilibrium can't and yaw angle. Keiichiro researched on the art anti-skid control that prevents the wheel slip of electrical motive unit based on the maximization of the tangential force for the motor vehicle. Therefore, the study failed to include the centrifugal force during train negotiating a curvature and the lateral sliding of the wheel form the yaw angle[11].

There is also a designed anti-skid control system used the anti-skid devices. The working principle of the main device needs the inputs parameters to provide the outputs signal which requires controlling the skidding. The slip rate, deceleration and speed difference are the main inputs of that device to be activated. The vehicle forward speed together with wheel speed would be analyzed during system processing step. The forward speed of the train is different of wheel speed because of electrical motor conversion and other rail/wheel contact parameters[12] and[13]. The adhesion force depends to the wheel rail contact parameters and during braking application. There is a little change according to the different inputs to the contact patch. The simulation was done in MATLAB software with the initial velocity of $80 \mathrm{~km} / \mathrm{h}$ to solve the re-adhesion of railway vehicles[14]. Therefore, this control was efficacy to solve the skidding problem on straight track and considering the only longitudinal slip/creep during braking application.

In order to improve the control system of the wheel sliding for high operation safety of the railway vehicle, the anti-skid braking control in curved track should be developed. Vehicle negotiating a curve the probability of derailment is high from lateral and longitudinal sliding which can cause the flange climbing. The lateral creep, longitudinal creep, the train forward velocity and the creep forces are considered as the main input of the system during simulation in MATLAB. In curved railroads, during an emergency stop and high deceleration the $\mathrm{ABS}$ allows the driver to apply the optimal braking force without locking or sliding the wheels. The controlled braking force helps to achieve the maximum available rail-wheel braking action with a prescribed adhesion mean in both lateral and longitudinal way so that the optimal/ short brake distance and curving performance can be achieved easily. This paper is structured in the following manner: after the introduction with the literature review, section two describe the wheel and rail contact in curved railroad and covering the analysis of the all contact parameters include creepages, yaw angle and lateral displacement and the creep forces, section three deal with the forces produced during braking and the working of ABS related to these forces, section four is where the simulation of the control system will be performed and the obtained results will be analyzed then the research will be end up by providing the conclusion part of the entire work. 


\subsection{Wheel and rail contact in curved track}

The wheelset of a railway vehicle during negotiating a curved track has three degrees of freedom from the yaw angle, lateral displacement and longitudinal motions. The wheelset is taken as a rigid material and attached to the bogie frame though different components[15]. It creates a moving line on a track a rotational speed that a vehicle should follow with its forward speed. However, the negotiation of a curved track by railway vehicle, the yaw motion or yaw angle plays an important role compared to the lateral displacement and longitudinal motions as shown in Figure1. The starting of curve, yaw dampers create a yaw angle which is the same to the attack angle and proportional to the equilibrium can't for better stability and ride comfort of a railway vehicle and negotiating a curvature in safety mean.

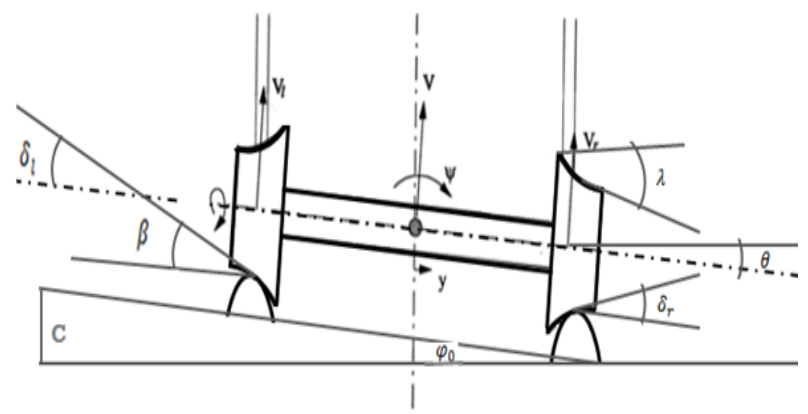

Figure 1. Geometry of a wheelset negotiating a curve

The figure 1 illustrates a wheelset negotiating a curve. The left wheel on the outer rail is relatively super elevated to the right wheel on the inner rail at angle $\left(\boldsymbol{\varphi}_{0}\right)$. The track has a right-handed curve and the wheelset has two degrees of freedom from yaw and lateral motion. The can't or super elevation $(\mathrm{C})$ together with wheel profile geometry caused the right wheel/rail contact with small radius compared to the left wheel because of their conical shape. For stability and ride comfort of a railway vehicle on a curved track, the lateral and yaw motion should be small as we compare to the longitudinal one but not negligible[16]. The speed of the wheelset $(\mathrm{V})$ in the curved track is defined by the three different variable velocities. There is yaw rate $(\dot{\psi})$ from yaw angle $(\psi)$, lateral velocity $(\dot{\boldsymbol{y}})$ from lateral displacement $(\mathrm{y})$ and spin speed $(\dot{\boldsymbol{\theta}})$ from pitch angle $(\theta)$ [17]. The left wheel with velocity $\left(V_{l}\right)$ has a long distance to move compare to the right wheel with velocity $\left(V_{r}\right)$. Therefore, the velocity different of the wheels during negotiating curves and the braking effects on the same axle causes some slippages of the wheels on rail in both lateral and longitudinal direction. The slip ratios of the wheels are the same as the creepages between wheel and rail contact in the area of contact patch.

\section{Creepages at the right wheel}

$$
\begin{aligned}
& \gamma_{x r}=\frac{1}{V}\left\{V\left(1-\left(\frac{a}{R}\right)-\left(\frac{r_{r}}{r_{0}}\right)\right)+a \dot{\psi}-r_{r} \dot{\theta}\right\} \\
& \gamma_{y r}=\sec \delta_{r}\left[\dot{y}-V \psi+r_{r} \dot{\beta}\right] \\
& \gamma_{\psi r}=\frac{1}{V}\left[\operatorname{Sin} \delta_{r}(\Omega+\dot{\theta})+\operatorname{Cos} \delta_{r}\left(\dot{\psi}-\frac{V}{R}\right)\right]
\end{aligned}
$$

\section{Creepages at left wheel}

$$
\begin{aligned}
& \gamma_{x l}=\frac{1}{V}\left\{V\left(1+\left(\frac{a}{R}\right)-\left(\frac{r_{l}}{r_{0}}\right)\right)-a \dot{\psi}-r_{l} \dot{\theta}\right\} \\
& \gamma_{y l}=\sec \delta_{l}\left[\dot{y}-V \psi+r_{l} \dot{\beta}\right] \\
& \gamma_{\psi l}=\frac{1}{V}\left[-\operatorname{Sin} \delta_{l}(\Omega+\dot{\theta})+\operatorname{Cos} \delta_{l}\left(\dot{\psi}-\frac{V}{R}\right)\right]
\end{aligned}
$$

The constraints equations of creepages of wheel/rail contact on a curved track depend to the different parameters/variables include: yaw angle, forward velocity, the contact angle, radius of curvature, super elevation, rolling angle, wheelset lateral displacement, and pitch angle. The all variables are easy to be determined except yaw angle. Yaw angle can be calculated with the analytical method from the wheelset dynamic equations based on the lateral input force and yaw input moment[18].

$$
\begin{aligned}
& m \ddot{y}+\frac{2 f_{22} \dot{y}}{V}+K_{y} y-2 f_{22} \psi \\
& +\left(\frac{2 f_{23}}{V}-\frac{I_{y} K V}{r_{0} l}\right) \dot{\psi}=-\frac{m V^{2}}{R_{0}}+m g \varphi_{0}+\frac{\sigma I_{y} V^{2}}{R_{0} r_{0}}+\frac{2 f_{23}}{R_{0}} \\
& \quad \frac{2 f_{11} \lambda_{0} l y}{r_{0}}-\left(\frac{2 f_{23}}{V}-\frac{I_{y} \delta_{0} V}{r_{0} l}\right) \dot{y}+I_{z} \ddot{\psi} \\
& +\frac{2 f_{11} l^{2} \dot{\psi}}{V}+K_{\psi} \psi=\frac{I_{y} V \dot{\varphi}_{0}}{r_{0}}-I_{z} V S R_{0}-\frac{2 f_{11} l^{2}}{R_{0}}
\end{aligned}
$$

Solving the equations (7) and (8) using the state space method

$$
\left[\begin{array}{cc}
m & 0 \\
0 & I_{z}
\end{array}\right]\left\{\begin{array}{c}
\ddot{y} \\
\ddot{\psi}
\end{array}\right\}+\left[\begin{array}{cc}
\frac{2 f_{22}}{V} & \left(\frac{2 f_{23}}{V}-\frac{I_{y} K V}{r_{o} l}\right) \\
-\left(\frac{2 f_{23}}{V}-\frac{I_{y} \delta_{0} V}{r_{0} l}\right) & \frac{2 f_{11} l^{2}}{V}
\end{array}\right]\left\{\begin{array}{l}
\dot{y} \\
\dot{\psi}
\end{array}\right\}
$$

$$
\begin{aligned}
& +\left[\begin{array}{cc}
K_{y} & -2 f_{22} \\
\frac{2 f_{11} \lambda_{0} l}{r_{0}} & K_{\psi}
\end{array}\right]\left\{\begin{array}{l}
y \\
\psi
\end{array}\right\} \\
& =\left\{\begin{array}{c}
-\frac{m V^{2}}{R_{0}}+m g \varphi_{0}+\frac{\sigma I_{y} V^{2}}{R_{0} r_{0}}+\frac{2 f_{23}}{R_{0}} \\
\frac{I_{y} V \dot{\varphi}_{0}}{r_{0}}-I_{z} V S R_{0}-\frac{2 f_{11} l^{2}}{R_{0}}
\end{array}\right\} \\
& {[M] \ddot{X}+[C] \dot{X}+[K] X=F}
\end{aligned}
$$

Where:

$$
\begin{aligned}
& \ddot{\mathbf{X}}=\left\{\begin{array}{c}
\ddot{y} \\
\ddot{\psi}
\end{array}\right\} \quad \dot{\mathbf{X}}=\left\{\begin{array}{c}
\dot{y} \\
\dot{\psi}
\end{array}\right\} \quad \mathbf{X}=\left\{\begin{array}{c}
y \\
\psi
\end{array}\right\} \\
& {[\boldsymbol{M}]=\left[\begin{array}{cc}
m & 0 \\
0 & I_{z}
\end{array}\right]} \\
& {[C]=\left[\begin{array}{cc}
\frac{2 f_{22}}{V} & \left(\frac{2 f_{23}}{V}-\frac{I_{y} K V}{r_{o} l}\right) \\
-\left(\frac{2 f_{23}}{V}-\frac{I_{y} \delta_{0} V}{r_{0} l}\right) & \frac{2 f_{11} l^{2}}{V}
\end{array}\right]}
\end{aligned}
$$




$$
\begin{aligned}
& {[\boldsymbol{K}]=\left[\begin{array}{cc}
K_{y} & -2 f_{22} \\
\frac{2 f_{11} \lambda_{0} l}{r_{0}} & K_{\psi}
\end{array}\right]} \\
& {[\boldsymbol{F}]=\left[\begin{array}{c}
-\frac{m V^{2}}{R_{0}}+m g \varphi_{0}+\frac{\sigma I_{y} V^{2}}{R_{0} r_{0}}+\frac{2 f_{23}}{R_{0}} \\
\frac{I_{y} V \dot{\varphi}_{0}}{r_{0}}-I_{z} V S R_{0}-\frac{2 f_{11} l^{2}}{R_{0}}
\end{array}\right]}
\end{aligned}
$$

The nonlinear system of equation we have is solved with the help of state space model have separated into four dependent variables ( $\mathrm{M}, \mathrm{C}, \mathrm{K}$ and $\mathrm{F})$. The transformation of normal equation into state space equations, the below state variables have chosen:

$$
\begin{gathered}
Z=\left\{\begin{array}{l}
Z_{1} \\
Z_{2}
\end{array}\right\} \\
Z_{1}=\{X\} \\
Z_{2}=\{\dot{X}\} \\
\dot{Z}_{1}=Z_{2} \\
\dot{Z}_{2}=\{\ddot{X}\} \\
\dot{Z}=\left\{\begin{array}{l}
\dot{Z}_{1} \\
\dot{Z}_{2}
\end{array}\right\}
\end{gathered}
$$

The state space standard form is use to write the system equation in summarized manner

$$
\begin{aligned}
\dot{Z} & =A Z+B U \\
y & =C Z+D U \\
A & =\left[\begin{array}{cc}
0_{2,2} & I_{z_{2,2}} \\
-M^{-1} K & -M^{-1} C
\end{array}\right] \\
B & =\left[\begin{array}{c}
0_{2,2} \\
M^{-1}
\end{array}\right] \\
U & =F
\end{aligned}
$$

In our case we assume that, $\mathrm{C}=1$

$$
\mathrm{D}=0
$$

Where:

\section{$\mathrm{Z}=$ State vector}

$\dot{Z}$ Derivative of the state vector with respect to time

$$
\mathrm{Y}=\text { Output vector }
$$

$\mathrm{U}=$ Input or control vector

$$
\mathrm{A}=\text { Amplification matrix }
$$

$\mathrm{B}=$ Actuation matrix

\section{$\mathrm{C}=$ Measurement matrix}

\section{$\mathrm{D}=$ Feedforward matrix}

From matrix formed by the equations (7) and (8), rearranging equation of yaw input moment and lateral displacement. Therefore, the boundary conditions have settled in line with equations aspect and we solve for yaw angle $(\psi)$ and lateral displacement (y). The constant parameter is the curve length of the track. Both the yaw angle and the lateral displacement are changing in function with the constant va- riable. The railway vehicle is yawing at a small angle in one direction with the help of the rotating table at the vehicles junction. From the straight track to the curvature, there is an attack angle which causes the yaw angle gets higher to follow the curve with the help of the yaw dampers. The two yaw dampers have the different positions according to the direction of the curvature. The Figure 2 shows the left curve negotiation hence at the starting the left damper retracts and the right damper extends. Within a certain moving of the railway vehicle in a curved track line and the effect of curve length cause the two dampers to change position. However, the yaw dampers back to the steady state after the curvature and let the vehicle to move in straight mean and here the lateral suspension are in static state. The lateral displacement helps the axle of the wheelset to flow the yaw angle and the track line and let the left and right wheel rotate at different speed with different radius at the contact patch.

Therefore, the yaw angle is lagging to the lateral displacements of the whole wheelsets of bogie by lateral suspension with respect to the direction of curvature. The moving of vehicle in straight line doesn't affect the lateral suspension and its yawing is at a static state with zeroed angle. The angle increasing to negotiate a curve in different direction according to the curve geometry and after a certain period of time it turns to its original position when the vehicle is out of the curvature as it is shown in Figure 4.

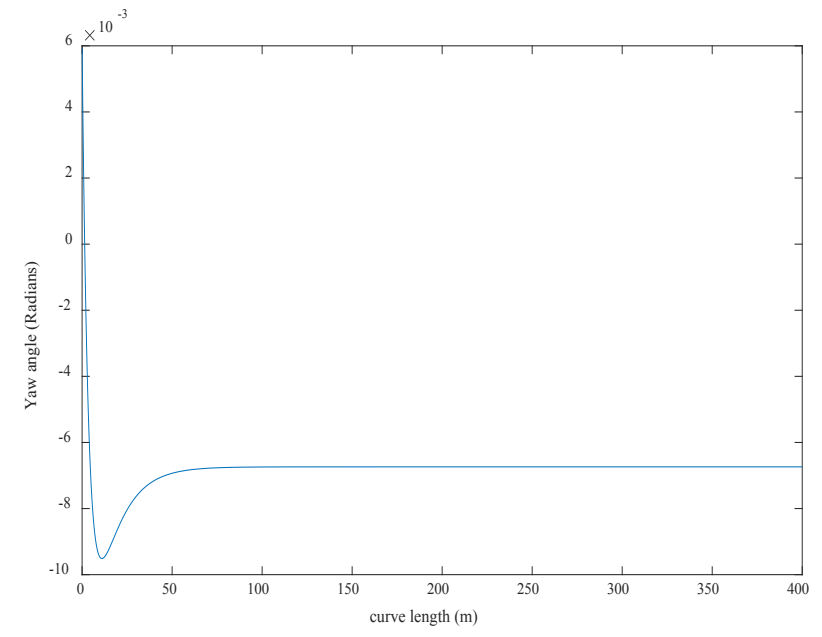

Figure 2. The yaw angle with respect to curve length

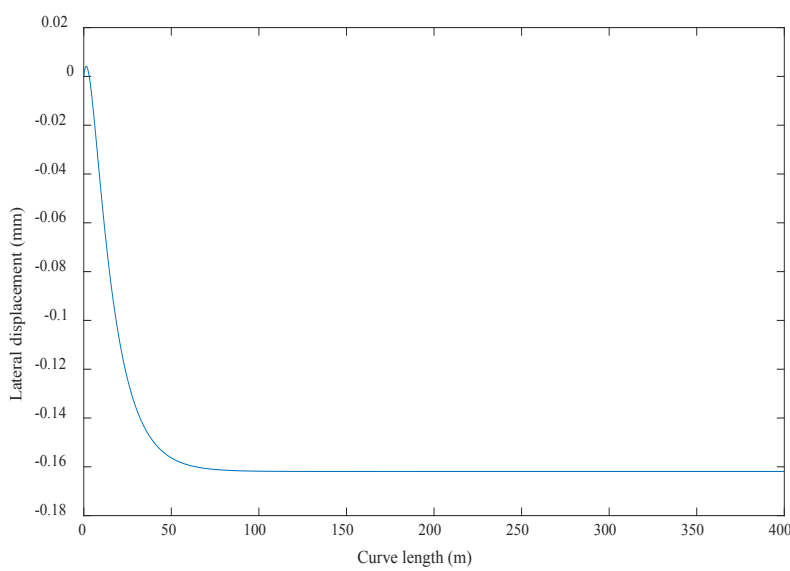

Figure 3. The lateral displacement with respect to curve length 


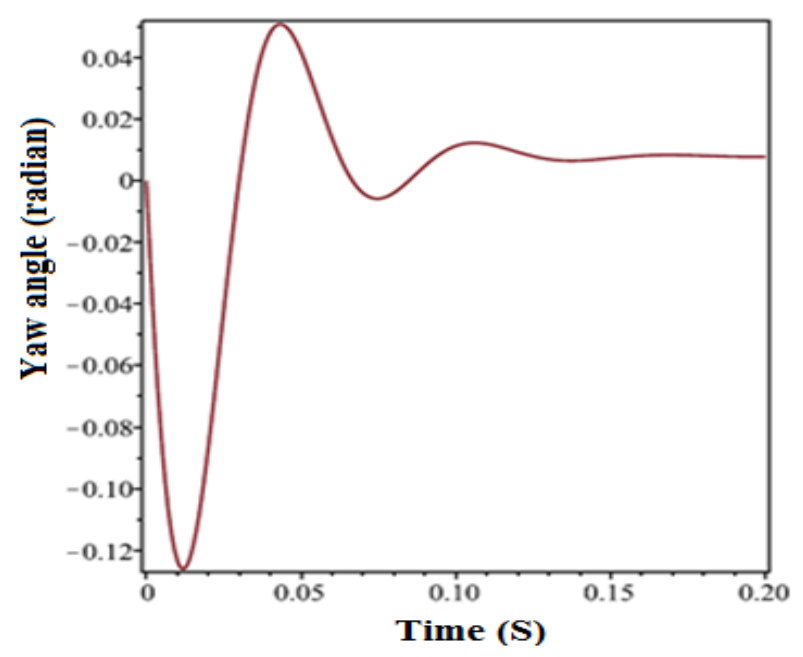

Figure 4. Yaw angle $(\psi)$ versus time

The negotiation of curve by a train at the starting the effects of curve radius and super elevation play an important role to the lateral suspension and yaw angle. The yaw angle is too high from zero and the change of lateral displacement is accelerated from the steady state of the wheelset as shown in yellow color for unstable of wheelset to change the straight track to the curved track with an attack angle. The rotating table with its two suspension yaw dampers one extends and other retract to negotiate the curve cause the negative and the positive yaw angles from the right wheels and the left wheels as shown in Figure 4 at $\mathrm{Z}$ axis. The lateral displacement ( $X$ axis) comes from that wheelset axle which is not horizontal at both right and left side. The lateral displacements also change with respect to time and becomes to the initial state at the straight track after curve is negotiated. Then after some duration wheels follow a curving line created by the lateral suspension by the yaw angle. The steady state or unstable of the wheelset, the yaw angle and the degree of curvature are in correlation to let the train junctions work properly. The yaw angle and lateral displacement are varying based to the can't angle, speed of the train and the radius of curvature. Therefore, the normal can't angle helps in curve negotiation in equilibration of bogie weight and the total centrifugal forces hence all should be varying with time.

The surface plot in Figure 5 explains the contribution of equilibrium can't in curve negotiation of the wheel set. The same as the track of AALRT, some curvatures have the equilibrium cants which are below to the standard compared to their curve radiuses. However, at the low can't the yaw is high, which is harder for the rotating table to initiate the wheelset to start a curve by entering with an attack angle. The yaw angle and lateral displacement are exaggerated at the yellow color with the time change according to the curvature length. The graph also shows how a small radius of curvature causes the wheelset to negotiate curve hardly by the fluctuations looks in that surface graph. The assumption is that the wheel is a rigid body with a constant radius. While, in reality it's different because at the contact patch there is some deformation from different parameters and the forces applied to it. The normal forces of the contact patch are perpendicular to the contact plane and have both lateral and vertical direction with different magnitude. However, the friction between wheel and rail when the train is in motion or stop on curved track is linearly depend on the normal forces, braking forces, lateral and longitudinal friction forces. Therefore, there is no difference between resultant friction force and resultant creep force at the contact patch. They are almost equal in magnitude on each wheel/rail interface.

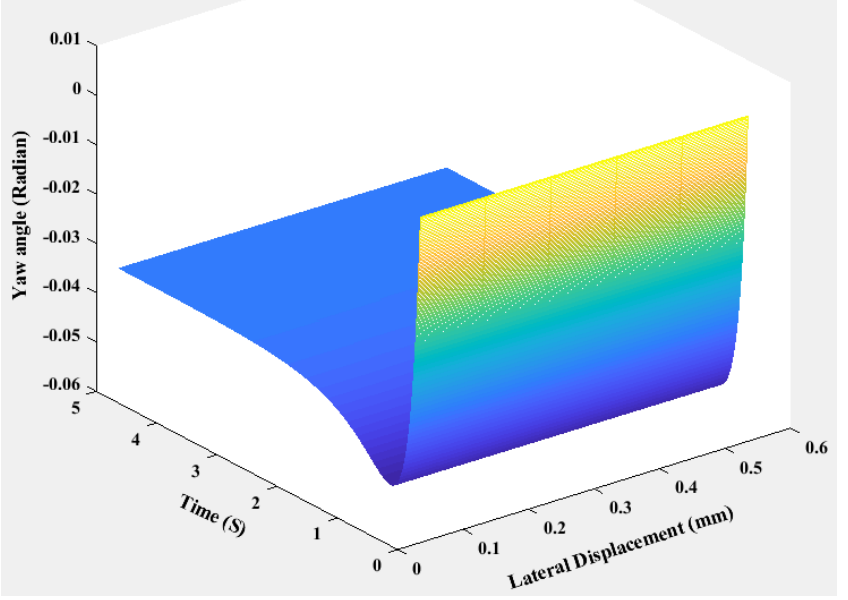

Figure 5. Curve of yaw angle with respect to cant and time

\section{The creep forces}

$$
\begin{aligned}
& F_{\gamma X r}=-f_{33} \gamma_{x r}+\left(f_{11} \gamma_{y r}+f_{12} \gamma_{\psi r}\right) \cos \left(\delta_{r}-\beta\right) \psi \\
& F_{\gamma y r}=\left(-f_{33} \gamma_{x r}\right) \psi-\left(f_{11} \gamma_{y r}+f_{12} \gamma_{\psi r}\right) \cos \left(\delta_{r}-\beta\right) \\
& F_{\gamma X l}=f_{33} \gamma_{x l}+\left(f_{11} \gamma_{y l}+f_{12} \gamma_{\psi l}\right) \cos \left(\delta_{l}+\beta\right) \psi \\
& F_{\gamma y l}=-\left(f_{11} \gamma_{y l}+f_{12} \gamma_{\psi l}\right) \psi+\left(-f_{11} \gamma_{y l}-f_{12} \gamma_{\psi l}\right) \cos \left(\delta_{l}+\beta\right)
\end{aligned}
$$

\section{Normal forces}

$$
\begin{aligned}
& F_{Z l} \cos \left(\delta_{l}+\beta\right)=\frac{M_{\beta}^{*}+F_{Z}^{*}\left[a-r_{r} \tan \left(\delta_{r}-\beta\right)\right]}{2 a-r_{r} \tan \left(\delta_{r}-\beta\right)-r_{l} \tan \left(\delta_{l}+\beta\right)} \\
& F_{Z r} \cos \left(\delta_{r}-\beta\right)=\frac{-M_{\beta}^{*}+F_{Z}^{*}\left[a-r_{l} \tan \left(\delta_{l}+\beta\right)\right]}{2 a-r_{r} \tan \left(\delta_{r}-\beta\right)-r_{l} \tan \left(\delta_{l}+\beta\right)}
\end{aligned}
$$

Where:

$$
\begin{aligned}
& M_{\beta}^{*}=I_{w z}(\ddot{\beta}+\ddot{\alpha})-I_{w y}(\Omega+\dot{\theta})\left[\dot{\psi}-\frac{V}{R}\right]-a\left(F_{\gamma z l}-F_{\gamma z r}\right) \\
& -r_{r} F_{\gamma y r}-r_{l} F_{\gamma y l}+h W_{e x t}\left(\beta_{d}-\beta\right)-\left(M_{\gamma x l}+M_{\gamma x r}+M_{s x}\right) \\
& F_{z}^{*}=m\left[\ddot{z}+a \ddot{\alpha}+\frac{V^{2}}{R} \alpha\right]-F_{\gamma z r}-F_{\gamma z l} \\
& +\left(W+W_{e x t}\right)+\left(\frac{W_{e x t}}{g}\right)\left(\frac{V^{2}}{R}\right) \alpha-F_{S z}
\end{aligned}
$$

The region of the contact patch is created by the slip and the adhesion areas. The Anti-lock Braking system (ABS), its purpose is to minimize that slippage area. The slip talk about in this paper is creepage. The adhesion or friction force on the curved track has two components, one in longitudinal direction and another in lateral direction. And they are independent with the friction coefficient (). The acceleration and deceleration of the wheel on the rail depend on the resultant friction force and the total mass applied on the con- 
tact patch.

$$
\begin{aligned}
& F_{X r}=F_{Z r} \cos \left(\delta_{r}-\beta\right) \mu_{x} \\
& F_{Y r}=F_{Z r} \cos \left(\delta_{r}-\beta\right) \mu_{y} \\
& F_{X l}=F_{Z l} \cos \left(\delta_{l}+\beta\right) \mu_{x} \\
& F_{Y l}=F_{Z l} \cos \left(\delta_{l}+\beta\right) \mu_{y} \\
& F_{R}=\sqrt{\left(F_{X r}\right)^{2}+\left(F_{Y r}\right)^{2}}=\sqrt{\left(F_{Z r} \cos \left(\delta_{r}-\beta\right) \mu_{x}\right)^{2}+\left(F_{Z r} \cos \left(\delta_{r}-\beta\right) \mu_{y}\right)^{2}} \\
& F_{L}=\sqrt{\left(F_{X l}\right)^{2}+\left(F_{Y l}\right)^{2}}=\sqrt{\left(F_{Z l} \cos \left(\delta_{l}+\beta\right) \mu_{x}\right)^{2}+\left(F_{Z l} \cos \left(\delta_{l}+\beta\right) \mu_{y}\right)^{2}} \\
& F_{c}=\frac{1}{R} \boldsymbol{m} V^{2}=\text { Sum all lateral force at the center gravity } \\
& \frac{d V_{l}}{d t}=\frac{2 F_{L}}{M A} \\
& \frac{d V_{r}}{d t}=\frac{2 F_{R}}{M A} \\
& \frac{d \omega_{l}}{d t}=\frac{1}{I w}\left(-T_{b}+F_{L} r_{l}\right) \\
& \frac{d \omega_{r}}{d t}=\frac{1}{I w}\left(-T_{b}+F_{R} r_{r}\right)
\end{aligned}
$$

\subsection{The Antilock System to braking force}

The force produced during braking is separated into two categories, disk-pads braking force and wheel-rail braking force. The source of the energy of the braking system of railway vehicle is compressed air. The brake system has many parts able to fulfill its function of converting kinetic energy of the vehicle into heat energy. During conversion, the direct contact parts are brake disk and brake pads. For the axle disk braking system, brake disk is fixed to the wheel axle and rotates at the same speed as the axle. The brake pads are two and fixed in a caliper then clamp the disk between them during braking. The force created between the pads and disk is called disk-pads braking force. However, this force increases as the pressure in the system increases. The disk-pads braking force is not enough to slow down or to stop a vehicle. Therefore, there is a force to be created between wheel and rail to suppress the friction or adhesion forces and it is called a wheel-rail braking force. The second force is not equal at both rear and front sides of the vehicle. During braking the weight of the vehicle is transferred from rear to front side that causes more load on the front side of the vehicle. The transfer of the vehicle weight from rear to front wheels depends on the length of the train and the bogie suspension.

The braking forces both disk-pad and wheel-rail braking forces are affected by the vehicle dynamics. The vehicle dynamics like stability, steering ability, ride comfort and better wheel-rail contact in a curved track should be maintained and the slip and locking up of wheels on the rail should be prevented by the ABS. During the contact of the wheel and rail, there is adhesion force which is equal or higher than the traction force. Excessive axle skids might cause undesirable wear and overheating of the rolling surface and the contact patch. The ABS modulates in real-time the motor drive torques in order to keep the axle skids within tolerable limits due to its function of reducing of braking force and wheel rotate again[19]. The torque from traction motor is transferred directly to the driving wheel axles, and the inertia of each wheel axle is increased compared to the transmission element inertia and the corresponding speed ratio.

$$
\boldsymbol{T}_{b}=(\boldsymbol{P} \times \boldsymbol{A} \times \boldsymbol{\mu} \times \boldsymbol{n}) \boldsymbol{R}_{d}
$$

\section{CONTROL SIMULATION AND ANALYSIS}

The control of antiskid braking system is an important subsystem of braking system that can reduce some accident in curved track. The wear and overheating between wheel and rail should also be avoided from the reduction of braking distance. The sliding and locking up of wheel on rail is reduced to the high level because the control of both longitudinal and lateral creepages. The locking up of the wheel is caused by the braking force application in system which is not proportion to the wheel rotation. The ABS as an electronic system can detect the lateral and longitudinal slippages or creepages of wheels and command automatically the braking pressure accordingly to be reduced for slip avoidance. During curve negotiation the speed of right and left wheels are different because of equilibrium can't, the different of chord to be travelled by wheels and effect of yawing from yaw angle. This modern antiskid control of the railway vehicle in curved track uses the all parameters of the vehicle during negotiating a curvature. The figure below shows the effect of antiskid control system on both wheel speed and the forward train speed.

The slippage of wheels in curvature shown in Figure 6 is a resultant of the lateral and longitudinal slip rates or creepages. The speed of $80 \mathrm{~km} / \mathrm{h}$ was the initial operation speed before driver applied on the brake level. The starting of braking operation, the disk-pads braking force is developed and the speed of wheel and train being reduced but with zero creepages of both lateral and longitudinal direction. However, at $70 \mathrm{~km} / \mathrm{h}$ of the train speed the wheel-rail braking force have been developed to continue slowing down the both speeds. Due to wheel-rail barking force is applied in the braking system, the resultant creepage/slip increases and here it is at 0.18 . The increasing of the slipping between the wheel and rail can't go beyond one. The antilock braking system can't allow the locking up of the wheels on the rail because its working is to create the close and open of the braking pressure by the bang- bang controller. The slip is represented by the sinusoidal line of up and down from the open close of the system. The speeds continue decreasing without continuous slippage. However, between 13 and 14 second the wheel rotation speed decreases at zero but the train speed decreases at 1.71 . This different is caused the wheel-rail braking force created. The train speed comes from the linear speed from the slippage of the wheel on the rail which takes few second to stop at the full stop of the slippage. Therefore, the braking system with the antiskid control system in curvatures does not present the locking up of the wheel on the rail. The stopping distance added after the nonrotating 
of the wheel is calculated in 0.5 second with speed below $0.855 \mathrm{~km} / \mathrm{h}$

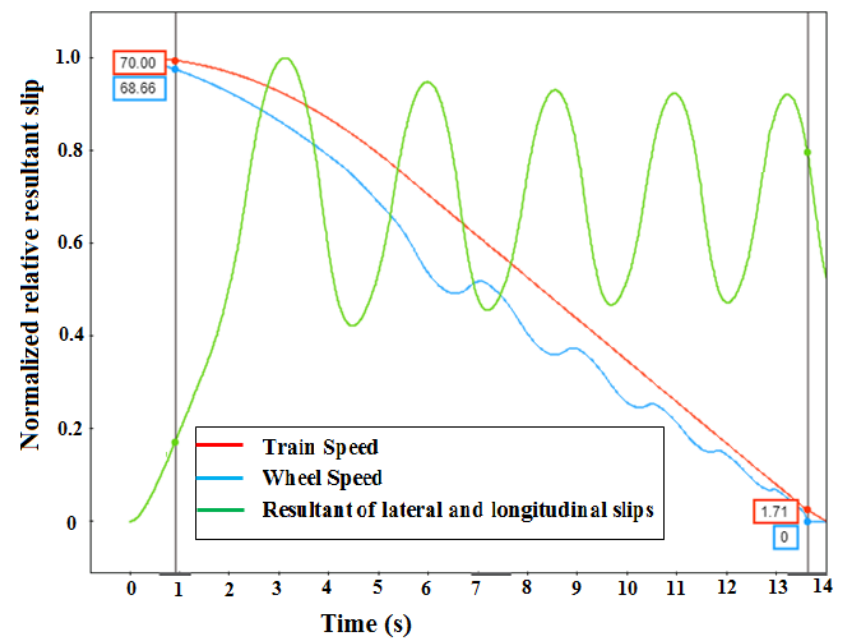

Figure 6. Slowdown of wheelset speed and train speed in curved track

\section{CONCLUSION}

In this paper, the study on railway vehicle negotiating curve principle and modelling and simulation of antiskid control system had been carried out. The simulation uses the results have been find out during modelling parts. The modeling covers the determination of the creepages, creep forces, braking forces. However, the creepages and creep forces produced during braking in curvatures have determined based on various curved track conditions and vehicle dynamics. The braking forces have determined based on the brake pressure to become the inputs parameter of the bang-bang controller during simulation. Based on the obtained result, to fight for safety in curved track of AARLT line, the antiskid control system needs to be applied in the braking system. Besides, control of longitudinal and lateral skidding of the wheels on the rail reduce the cost of maintenance of the wheel and rail. The Simulink modelling results have been validated by using the normal ABS. However, the normal ABS deals with the skidding in longitudinal means and this studied ABS discussed in this paper combines both longitudinal and lateral skidding. The method presented in this paper can be applied for modeling the active safety system of the railway vehicle negotiating a curvature and wheel tread wears reduction of the vehicle using the sharped curves. It is highly recommended to add the antiskid control system in the entire braking system of the railway vehicles used the track line have the sharp curvatures. The AALRT needs to use this system to prevent the crushing of wheel flange caused by the lateral skidding during brake application. The performed future works are the implementation of the system in the railway vehicle and additional of indicators in the dashboard to defined the status working operation of the system.

\section{REFERENCES}

[1] Ahmad, F., Hudha, K. Rivai. A., Zakaria, MMN (2008). Modeling and validation of vehicle dynamic performance in longitudinal direction. International Journal of Vehicle System Modelling and Testing, 5(4), 312-346
[2] Tudor, A., Sandu, N., Tountas, E. (2009). Wheel/rail friction power in curved track. U.P.B. Sci. Bull., Series D, 71(3): 75-88.

[3] B. Allotta, L. P. (25-29 May, 2010). Mutual interaction of parrallel connected induction motors on degraded adhesion conditions. The first joint international conference on multibody system dynamics (p. 4). Lappeenranta: Lappeenranta, Finiland.

[4] Uyulan, C., Gokasan, M., Bogosyan, S. (2017). Modeling, simulation and slip control of a railway vehicle integrated with traction power supply. Cogent Engineering, 4(1), 1312680.

[5] Choi, J. J., Park, S. H., Kim, J. S. (2007). Dynamic adhesion model and adaptive sliding mode brake control system for the railway rolling stocks. Proceedings of the Institution of Mechanical Engineers, Part F: Journal of Rail and Rapid Transit, 221(3), 313-320.

[6] Dukkipati, V. G. (1984). Dynamics of Railway Vehicle. Toronto: Toronto Orlando San Diego New York.

[7] Hur, M.-S. K.-M. (2014). Braking/Traction Control Systems of a Scaled Railway Vehicle. Proceedings of the 9th WSEAS International Conference on Robotics, Control and Manufacturing Technology, 1.

[8] Hur, M.-S. K.-M. (2017). Braking/Traction Control Systems of a Scaled Railway Vehicle for the Active Steering Testbed. Proceedings of the 9th WSEAS International Conference on Robotics, Control and Manufacturing Technology (pp. 74-79). 360-1 Woram-dong, Uiwang-si, Kyonggi-do KOREA: http://www.krri.re.kr.

[9] Srivastava, J. P., Sarkar, P. K., \& Ranjan, V. (2013, December). An approximate analysis for Hertzian elliptical wheel-rail contact problem. In Proceedings of the 1st International and 16th National Conference on Machines and Mechanisms (iNaCoMM2013) (pp. 249-253).

[10] Park, J. H., Koh, H. I., Hur, H. M., Kim, M. S., \& You, W. H. (2010). Design and analysis of an active steering bogie for urban trains. Journal of Mechanical Science and Technology, 24(6), 1353-1362.

[11] Wang, K., Huang, C., Zhai, W., Liu, P., \& Wang, S. (2014). Progress on wheel-rail dynamic performance of railway curve negotiation. Journal of traffic and transportation engineering (English edition), 1(3), 209-220.

[12] Kondo, K. (2012). Anti-slip control technologies for the railway vehicle traction. IEEE Vehicle Power and Propulsion Cconference (pp. 255-261). Seoul Olympic Parket: www.psma.com.

[13] Yang, L., Kang, Y., Luo, S., \& Fu, M. (2015). Assessment of the curving performance of heavy haul trains under braking conditions. Journal of Modern Transportation, 23(3), 169-175.

[14] Kim, M.S, Park, J.H., You, W.H. (2008). Construction of Active Steering System of the Scaled Railway Vehicle. International Journal Of Systems Applications, Engineering \& Development, 2(4): 217-226.

[15] Nejlaoui, M., Houidi, A., Affi, Z., \& Romdhane, L. (2012). A Critical Speed Optimization of Rail Vehicle System Based on Safety Criterion. In Condition Monitoring of Machinery in Non-Stationary Operations (pp. 201-211). Springer, Berlin, Heidelberg.

[16] Sahin, M., \& Samim Unlusoy, Y. (2010). Design and simulation of an ABS for an integrated active safety system for road vehicles. International journal of vehicle design, 52(1-4), 64-81.

[17] Soomro, Z. A. (2015). Correlation of Lateral and yaw analysis responses to tracking of linearlized rail wheelset model. Journal of Mechanical Engineering and Technology (JMET), 7(1).

[18] Mei, T. X., Yu, J. H., \& Wilson, D. A. (2008). A mechatronic approach for anti-slip control in railway traction. IFAC Proceedings Volumes, 41(2), 8275-8280.Tanelli, S. M. (2010). Active braking control sys- 
tems design for vehicles. Springer Science \& Business Media, 2.

[19] Wenliang Zhu1, a. F. (2016). Modeling and Anti-skid Control of the Rail Vehicle Braking System. 6th International Conference on Advanced Design and Manufacturing Engineering (ICADME 2016) (pp. 582-590). Shanghai, China: Atlantis Press.

[20] Wickens., A. (2005). Fundamentals of rail vehicle dynamics : guidance and stability. Lisse, The Netherlands: Swets \& Zeitlinger.

[21] Zhang Xiu-qin, Y. B.-n. (2012). ABS of Multi-axle Truck Based on ADMS/Car and Matlab/Simulink. SciVerse Science Direct Procedia Engineering, 120-124.

\section{APPENDIXES}

Table 1. The symbols and constants in equations

\begin{tabular}{|c|c|c|c|}
\hline $\mathbf{S} / \mathbf{N}$ & Symbol & & Meaning \\
\hline 1 & $\gamma_{x r}$ & \multicolumn{2}{|c|}{ Longitudinal creepage of the right wheel } \\
\hline 2 & $\gamma_{y r}$ & \multicolumn{2}{|c|}{ Lateral creepage of the right wheel } \\
\hline 3 & $\gamma_{x l}$ & \multicolumn{2}{|c|}{ Longitudinal creepage of the left wheel } \\
\hline 4 & $\gamma_{y l}$ & \multicolumn{2}{|c|}{ Lateral creepage of the left wheel } \\
\hline 5 & $\gamma_{\psi r}$ & \multicolumn{2}{|c|}{ Right wheel spinning } \\
\hline 6 & $\gamma_{\psi l}$ & \multicolumn{2}{|c|}{ Left wheel spinning } \\
\hline 7 & $F_{\gamma X r}$ & \multicolumn{2}{|c|}{ Longitudinal creep force of the right wheel } \\
\hline 8 & $F_{\gamma y r}$ & \multicolumn{2}{|c|}{ Lateral creep force of the right wheel } \\
\hline 9 & $F_{\gamma X l}$ & \multicolumn{2}{|c|}{ Longitudinal creep force of the left wheel } \\
\hline 10 & $F_{\gamma y l}$ & \multicolumn{2}{|c|}{ Lateral creep force of the left wheel } \\
\hline 11 & $F_{Z l}$ & \multicolumn{2}{|c|}{ Left wheel normal force } \\
\hline 12 & $F_{Z r}$ & \multicolumn{2}{|c|}{ Right wheel normal force } \\
\hline 13 & $F_{X r}$ & \multicolumn{2}{|c|}{ Longitudinal force on the contact patch at the right wheel } \\
\hline 14 & $F_{Y r}$ & \multicolumn{2}{|c|}{ Lateral force on the contact patch at the right wheel } \\
\hline 15 & $F_{X l}$ & \multicolumn{2}{|c|}{ Longitudinal force on the contact patch at the left wheel } \\
\hline 16 & $F_{Y l}$ & \multicolumn{2}{|c|}{ Lateral force on the contact patch at the left wheel } \\
\hline 17 & $F_{c}$ & \multicolumn{2}{|c|}{ Centrifugal force } \\
\hline 18 & $F_{R}$ & \multicolumn{2}{|c|}{ Right wheel contact patch resultant force } \\
\hline 19 & $F_{L}$ & \multicolumn{2}{|c|}{ Left wheel contact patch resultant force } \\
\hline 20 & $T_{b}$ & \multicolumn{2}{|c|}{ Braking torque } \\
\hline 21 & A & \multicolumn{2}{|c|}{ Pad area } \\
\hline 22 & $\mathrm{P}$ & \multicolumn{2}{|c|}{ Braking pressure } \\
\hline 23 & $\mathrm{n}$ & \multicolumn{2}{|c|}{ Number of pads } \\
\hline 24 & M & \multicolumn{2}{|c|}{ Mass matrix } \\
\hline 25 & $\mathrm{D}$ & \multicolumn{2}{|c|}{ Damping matrix } \\
\hline 26 & $\mathrm{~K}$ & \multicolumn{2}{|c|}{ Stiffness matrix } \\
\hline 27 & $\mathrm{~F}$ & \multicolumn{2}{|c|}{ Force matrix } \\
\hline $\mathbf{S} / \mathbf{N}$ & Symbol & Value & Meaning \\
\hline 28 & G & $1.435 \mathrm{~m}$ & The track gauge \\
\hline 29 & $\mathrm{a}$ & $0.7175 \mathrm{~m}$ & $\begin{array}{l}\text { The halftrack gauge or half of the distance } \\
\text { between contact pints on two rails }\end{array}$ \\
\hline 30 & $\mathrm{R}_{0}$ & $300 \mathrm{~m}$ & The radius of curvature \\
\hline 31 & $\mathrm{~m}$ & $1250 \mathrm{~kg}$ & Mass of the wheelset axle \\
\hline 32 & $\mathrm{~g}$ & $9.81 \mathrm{~N} / \mathrm{m}^{2}$ & Acceleration due to gravity \\
\hline 33 & $\mathrm{I}_{2}$ & $688 \mathrm{Kgm}^{2}$ & Roll moment of inertia of the wheelset \\
\hline 34 & $I_{w}$ & $688 \mathrm{Kgm}^{2}$ & Yaw moment of inertia of the wheelset \\
\hline 35 & $\mathrm{I}_{y}$ & $100 \mathrm{Kgm}^{2}$ & Spin moment of inertia of the wheelset \\
\hline 36 & $r_{0}$ & $0.42 \mathrm{~m}$ & Nominal radius of the wheel \\
\hline 37 & V & $17 \mathrm{~m} / \mathrm{s}$ & Axle speed \\
\hline 38 & $\mathrm{f} 11$ & $7.44 \mathrm{e} 6 \mathrm{~N}$ & Lateral creep force coefficient \\
\hline
\end{tabular}

\begin{tabular}{|c|c|c|l|}
\hline 39 & $f 12$ & $3120 \mathrm{Nm}^{2}$ & Lateral/spin creep force coefficient \\
\hline 41 & $\mathrm{f} 22$ & $6.79 \mathrm{e} 6 \mathrm{~N}$ & Spin creep force coefficient \\
\hline 41 & $\mathrm{f} 23$ & $13.7 \mathrm{e} 3 \mathrm{Nm}$ & spin creep force coefficient \\
\hline 42 & $\mathrm{f} 33$ & $2.563 \mathrm{e} 6 \mathrm{~N}$ & Longitudinal creep force coefficient \\
\hline 43 & $\mathrm{k}_{\mathrm{y}}$ & $23 \mathrm{e} 4 \mathrm{~kg} / \mathrm{m}$ & Lateral stiffness \\
\hline 44 & $\mathrm{c}_{\mathrm{y}}$ & $0 \mathrm{kgsec} / \mathrm{m}$ & Lateral damping \\
\hline 45 & $\mathrm{k}_{\psi}$ & $\begin{array}{c}25 \mathrm{e} 5 \mathrm{kgm} / \\
\mathrm{rad}\end{array}$ & Yaw stiffness \\
\hline 46 & $\mathrm{c}_{\psi}$ & $0 \mathrm{gmsec} / \mathrm{rad}$ & Yaw damping \\
\hline 47 & $\sigma=\delta_{0}$ & $0.0493 \mathrm{rad}$ & Initial tapper angle \\
\hline 48 & $\lambda$ & $0.05 \mathrm{rad}$ & Conicity angle \\
\hline 49 & WA or No & $12262.5 \mathrm{~N}$ & Axle load \\
\hline
\end{tabular}

